\title{
L-Menthol sprayed on gastric mucosa causes edematous change
}

Authors

Institution
Akihiro Mori, Hiroki Hachiya, Takayuki Yumura, Shun Ito, Shintaro Hayashi, Masashi Nozaki, Atsui Yoshida, Noritsugu Ohashi

Ichinomiya Nishi Hospital - Gastroenterology, Ichinomiya, Japan submitted 9. January 2014 accepted 28. February 2014

\section{Bibliography \\ DOI http://dx.doi.org/ \\ 10.1055/s-0034-1377172 \\ Published online: 23.6.2014 \\ Endoscopy International Open \\ 2014; 02: E51-E57 \\ (c) Georg Thieme Verlag KG \\ Stuttgart $\cdot$ New York \\ E-ISSN 2196-9736}

Corresponding author

\section{Akihiro Mori}

1 Kaimei-hira Ichinomiya Aichi

Japan

Ichinomiya 494-001

Japan

Fax: +81-586-48-0038

a-mori@anzu.or.jp
Background and study aims: L-Menthol (LM), sprayed on the distal gastric mucosa, is a safe antispasmodic agent used during esophagogastroduodenoscopy (EGD). However, it seems to affect gastric mucosal endoscopic findings. Therefore, we evaluated whether LM causes specific changes and impacts the endoscopic morphology of gastric lesions.

Patients and methods: A total of 98 patients scheduled to undergo EGD were randomly assigned to receive LM solution ( $160 \mathrm{mg}$ of $0.8 \% \mathrm{LM}$ added to $2.5 \mathrm{~mL}$ of indigo carmine [IC]; $n=49$; LM group) or decuple-diluted IC solution without LM ( $n=49$; placebo group). We compared the incidence of specific mucosal changes and the difference in the endoscopic findings of several gastric lesions between these groups.

Results: Annular-reticular - like mucosal changes appeared immediately after the administration of LM solution. This change was observed in

\section{Introduction}

\section{$\nabla$}

The early detection and treatment of gastric cancer are necessary to reduce mortality [1], although surgery is the standard treatment. Esophagogastroduodenoscopy (EGD) is useful for the detection of early gastric cancer because this technique can identify superficial flat and nonulcerative lesions. The suppression of gastric peristalsis promotes the detection of smaller lesions because it facilitates EGD [2,3]. Thus, antispasmodic agents such as butyl scopolamine bromide and glucagon are often injected before EGD to suppress gastric peristalsis. However, complications associated with these antispasmodic agents, including cardiovascular events and reactive hypoglycemia, have been reported $[4,5]$. Therefore, endoscopists should consider these complications, particularly in elderly patients.
The intragastric administration of L-Menthol (LM) was found to reduce gastric spasm during
$71.4 \%$ of the LM group compared with $12.2 \%$ of the placebo group $(P<0.01)$. In the placebo group, this change was observed in $14.7 \%$ of subjects with atrophic gastritis compared with $6.7 \%$ of those without atrophic gastritis $(P=0.39)$, whereas in the LM group, this change was observed in $84.8 \%$ of subjects with atrophic gastritis compared with $43.8 \%$ of those without atrophic gastritis $(P<0.01)$. Most early gastric cancers, erosions, and ulcers observed in this study became well demarcated after LM administration, although the incidence of gastric lesions did not differ significantly between the two groups.

Conclusion: LM changes the gastric mucosa into edematous mucosa, and this occurs more frequently in atrophic gastric mucosa than in pathologic lesions. LM may facilitate the demarcation of pathologic gastric lesions without intestinal metaplasia.

EGD and to be more effective than the intramuscular injection of butyl scopolamine bromide $[2,6]$. The use of LM as an alternative antispasmodic agent would increase the safety of EGD, even in elderly patients [7], with a possibly better detection of gastric lesions. We have therefore used LM as an antispasmodic agent. On one occasion, however, we noted that directly spraying LM onto the distal stomach caused a specific endoscopic mucosal change - namely, annular and/or reticular edematous thickening - just after administration ( $\bullet$ Fig. 1, Fig.2). The change seemed to occur mainly in mucosa affected with atrophic gastritis. Hikichi and colleagues reported that the intragastric administration of LM emphasized the margin of gastric cancer because the mucosa around the tumor became edematous [8]. However, limited information is available regarding the impact of LM on the gastric mucosa. The aim of the present study therefore was to evaluate whether LM causes specific gas- 

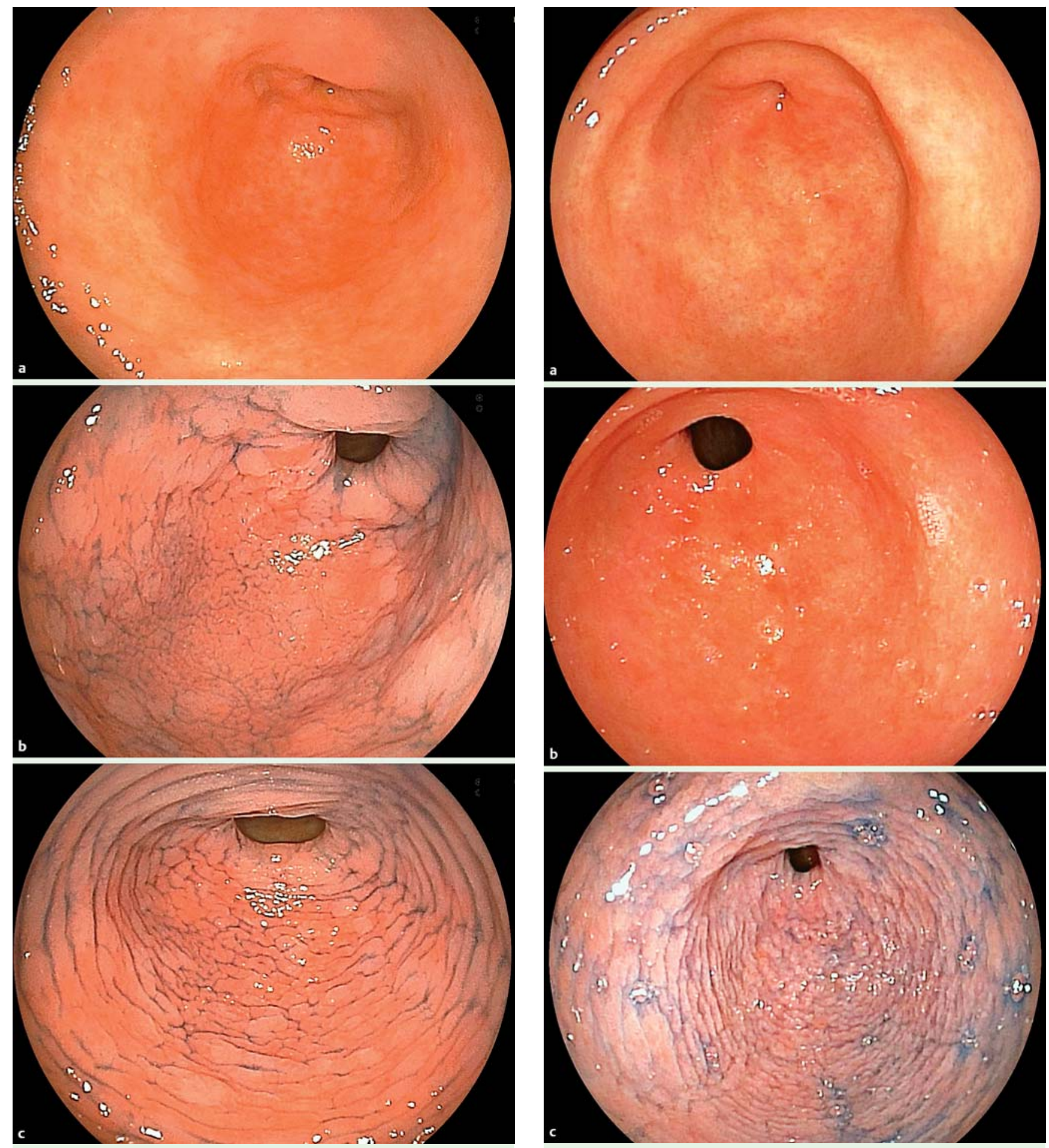

Fig. 1 Endoscopic views showing the distal gastric mucosa. a Before spraying either indigo carmine solution or L-Menthol (LM). b After spraying only indigo carmine solution. c After spraying a mixed solution of indigo carmine and LM.

tric mucosal changes and whether atrophic gastritis is associated with this change. In addition, we investigated how LM impacts the endoscopic morphology of gastric lesions.

Fig.2 Endoscopic views showing the distal gastric mucosa. a Before spraying either indigo carmine solution or L-Menthol (LM) solution. b After spraying only LM. c After spraying only indigo carmine solution subsequent to the administration of LM.

\section{Methods}

$\nabla$

\section{Patients}

Consecutive patients who were scheduled to undergo EGD screening or follow-up for upper gastrointestinal disease from 4 June to 31 July 2013 were enrolled in this study. The exclusion criteria were previous surgery involving the upper gastrointestinal tract, upper gastrointestinal bleeding requiring hemostasis, 
scheduled endoscopic surgery, and a history of hypersensitivity to LM or peppermint oil. In addition, at the initial endoscopic observation, subjects with a poor endoscopic view due to gastric residual or with signs of annular-reticular - like mucosal changes were excluded. This study was registered in a clinical trial database on 3 June 2013 (University Hospital Medical Information Network: UMIN000010859) and was approved by the hospital review board for human research. All patients provided written informed consent before participating in this study.

\section{Procedures}

This was a single-center, prospective, randomized, open-label, placebo-controlled study. Because the investigator was often able to distinguish placebo-treated patients by the aroma present at the time of LM administration despite wearing gloves and a mask, this study failed to fulfill the criteria for a double-blinded trial. The subjects were randomly allocated to the LM group or placebo group by the envelope method before EGD. To dissolve the stomach mucous layer, each subject ingested a solution containing $20000 \mathrm{U}$ of pronase (Kaken Pharmaceutical Co., Ltd., Tokyo, Japan), $5 \mathrm{~mL}$ of dimethicone, and $1 \mathrm{~g}$ of $\mathrm{NaHCO}_{3}$ in $80 \mathrm{~mL}$ of water 10 minutes before EGD. All procedures were performed without the administration of any antispasmodic agents or sedative agents except LM. An ultrathin endoscope (EG580NW; FUJIFILM, Tokyo, Japan) was used with a transnasal approach in all subjects. Topical anesthesia of the nasal cavity was performed by spraying $0.111 \%$ tramazoline hydrochloride and embrocation of $8 \%$ lidocaine liquid with $10 \mathrm{Fr}$ and $18 \mathrm{Fr}$ catheters [9]. To enhance the minimal changes of the gastric mucosa, indigo carmine (IC) was mixed into both the LM and the placebo solutions. According to the results of a preliminary pilot study, the mucosal change that appeared with LM alone became sharper following the subsequent administration of IC alone ( $\bullet$ Fig.2). However, when we administered the mixed solution of IC and LM, the same change appeared ( Fig. $1 \mathrm{c}$ ). This change was not observed after IC solution had been sprayed alone ( $\bullet$ Fig. 1 b). In addition, bubbles caused by the administration of LM were often observed. Therefore, the LM solution was prepared by mixing $20 \mathrm{~mL}$ of $0.8 \%$ LM (160 mg) (Nihon Pharmaceuticals Co. Ltd., Tokyo, Japan), $0.5 \mathrm{~mL}$ of dimethicone, and $2 \mathrm{~mL}$ of IC. It was pharmacologically confirmed that the compounding variation of the LM solution had been stable for at least 6 hours. The placebo solution was prepared by mixing $20 \mathrm{~mL}$ of water, $0.5 \mathrm{~mL}$ of dimethicone, and $2 \mathrm{~mL}$ of IC. The concentration of IC in each solution was maintained at $10 \%$. Each solution was sprayed directly with a syringe onto the distal stomach via the biopsy channel of the endoscope. Residual liquid was pushed out with air. The solution was applied to the entire surface of the stomach via air suctioning. Routine endoscopic observation was repeated before and after the solution was administered. In cases in which gastric lesions were detected in the placebo group, after routine observation, the LM solution was resprayed on the lesion and the sequential endoscopic morphologic changes of the lesion were observed. In addition, early gastric cancers that were detected during the study were re-examined on another day to estimate the difference in fine structural changes of the early gastric cancers between the LM and placebo groups with a magnifying endoscope and blue laser imaging (EGL590ZW; FUJIFILM). All gastric lesions detected during the study were diagnosed histologically by biopsy.

Gastric peristalsis was evaluated before and after the solution was administered according to the partially modified Niwa classification $[2,3,10]$ : grade 1 (no peristalsis), grade 2 (mild peri- stalsis), grade 3 (moderate peristalsis), grade 4 (vigorous peristalsis), and grade 5 (markedly vigorous peristalsis). In this study, these classifications were used to divide patients into two groups: a peristaltic suppressed state group (i.e., grade 1 or 2 ) and a peristaltic state group (i.e., grade 3,4 , or 5 ).

\section{Evaluation of the effect of L-menthol on the gastric mucosa}

During routine endoscopic observation before the administration of each solution, the existence of pathologic lesions or atrophic gastritis was evaluated. Endoscopic findings of atrophic gastritis were defined as surface irregularity, visibility of the vascular pattern, patchy erythema [11], and the absence of a regular arrangement of collecting venules. Next, within 3 minutes of spraying each solution, routine endoscopic observation was repeated, and the endoscopic morphologic changes of the gastric lesion or the appearance of specific mucosal changes (annular-reticularlike change) was evaluated. The location of specific mucosal changes in the stomach was also identified.

One endoscopist (A. M.) performed all routine nasal endoscopies and detailed magnifying endoscopies in the present study, recording all endoscopic findings and the results of the gastric peristalsis evaluation.

\section{Outcomes}

The primary outcome was to evaluate whether LM causes the specific change and whether the atrophic gastritis is associated with this change. The secondary outcome was to investigate the impact of LM administration on the endoscopic morphology of gastric lesions and the antiperistaltic effect of the LM solution used in the study.

\section{Sample size and statistical analysis}

Before performing the study, we performed a preliminary trial. The incidence rates of the specific mucosal change in the LM and placebo groups were expected to be $70 \%$ (35 of 50 subjects) and $30 \%$ (15 of 50 subjects), respectively. The sample size required to detect a difference in incidence rates between the groups with a significance level of $5 \%$ (two-sided) and a power of $90 \%$ was calculated to be 36 per group by Fisher's exact test. We required 50 patients per group because of the concern that patients with a poor endoscopic view would be included.

Statistical analysis was performed with Welch's $t$ test, Fisher's exact probability test, and the chi-square test. All analyses were performed with the Statistical Package for the Social Sciences (SPSS) for Windows (SPSS Japan Inc., Tokyo, Japan). A $P$ value of less than 0.05 was considered significant.

\section{Results}

\section{$\nabla$}

\section{Study population}

A total of 100 subjects provided written consent and were enrolled in the study. Of these, 50 were randomly assigned to the LM group and 50 to the placebo group. One subject in each group showed annular-reticular - like mucosal changes at the initial endoscopic observation. These two subjects were excluded, resulting in 49 subjects enrolled in the LM group and 49 enrolled in the placebo group. All subjects underwent EGD without sedation successfully.

The baseline characteristics of the two groups are shown in - Table 1. No significant differences were found between the 
Table 1 Baseline characteristics.

\begin{tabular}{|c|c|c|c|}
\hline & $\begin{array}{l}\text { L-Menthol } \\
(n=49)\end{array}$ & $\begin{array}{l}\text { Placebo } \\
(n=49)\end{array}$ & \\
\hline Mean age, y (range) & $64(25-94)$ & $59(26-82)$ & n.s. \\
\hline Sex, male/female & $23 / 26$ & $29 / 20$ & n.s. \\
\hline $\begin{array}{l}\text { Anti-Helicobacter pylori IgG } \\
\text { antibody positive, No. (\%) }\end{array}$ & $26(53)$ & $26(53)$ & n.s. \\
\hline $\begin{array}{l}\text { After Helicobacter pylori } \\
\text { eradication, №. (\%) }\end{array}$ & $5(10)$ & $6(12)$ & n.s. \\
\hline \multicolumn{4}{|l|}{ Endoscopic findings, No. (\%) } \\
\hline Atrophic gastritis & $33(67)$ & $34(69)$ & n.s. \\
\hline Gastric adenoma & $1(2)$ & $1(2)$ & n.s. \\
\hline Early gastric cancer & $1(2)$ & $3(6)$ & n.s. \\
\hline Advanced gastric cancer & $1(2)$ & $0(0)$ & n.s. \\
\hline Gastric erosion & $8(16)$ & $12(24)$ & n.s. \\
\hline Gastric ulcer & $4(8)$ & $2(4)$ & n.s. \\
\hline Gastric ulcer scar & $2(4)$ & $1(2)$ & n.s. \\
\hline Hyperplastic polyp & $2(4)$ & $2(4)$ & n.s. \\
\hline
\end{tabular}

IgG, immunoglobulin G; n.s., not significant.

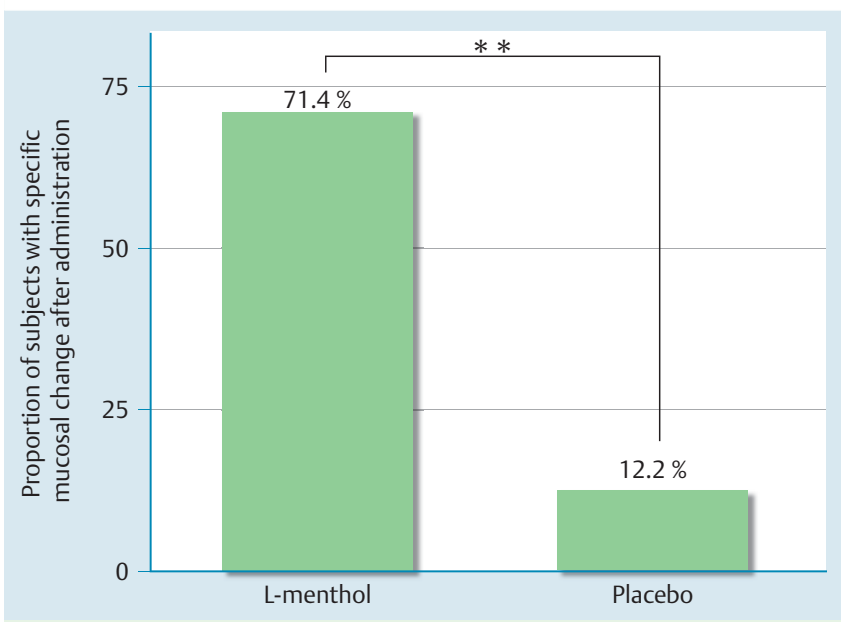

Fig. 3 The proportion of subjects with specific mucosal changes in each group. This proportion was significantly higher in the L-menthol group than in the placebo group $\left(^{* *}: P<0.01\right)$.

groups in regard to age, sex, anti-Helicobacter pylori immunoglobulin $\mathrm{G}(\mathrm{IgG})$ antibody positivity, and number of subjects who underwent $H$. pylori eradication.

\section{Primary outcome}

The proportion of subjects with specific mucosal changes after solution administration was significantly higher in the LM group (71.4\%, 35 of 49 subjects) than in the placebo group (12.2\%, 6 of 49 subjects; $P<0.01$ ) ( $\bullet$ Fig. 3 ). The changes were observed only in the distal stomach in the placebo group but spread to the gastric body in $54 \%$ ( 19 of 35 subjects) in the LM group. The 19 subjects with changes to the gastric body showed advanced atrophic gastritis. In the LM group, the proportion of patients with specific mucosal changes was significantly higher among subjects with atrophic gastritis ( $84.8 \%, 28$ of 33 subjects) than in those without (43.8\%, 7 of 16 subjects; $P<0.01$ ). In contrast, in the placebo group, the proportion of patients with specific mucosal changes was unaffected by the presence or absence of atrophic gastritis ( $14.7 \%, 5$ of 34 subjects vs. $6.7 \%, 1$ of 15 subjects; $P=0.39$ ) ( $\bullet$ Fig. 4). The histopathologic findings of the affected mucosa in-

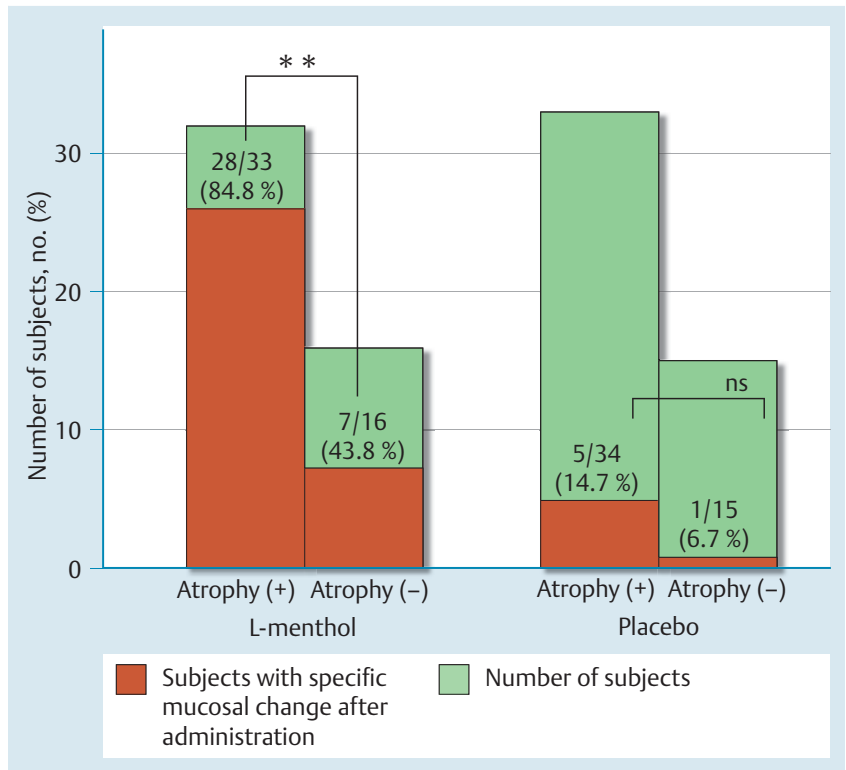

Fig. 4 The proportion of subjects with specific mucosal changes depended on the presence or absence of atrophic gastritis in each group. In the placebo group, this proportion did not depend on the presence or absence of atrophic gastritis $(P=0.39)$, whereas in the L-menthol group, it was significantly higher in subjects with atrophic gastritis than in those without atrophic gastritis $\left({ }^{* *}: P<0.01\right)$.

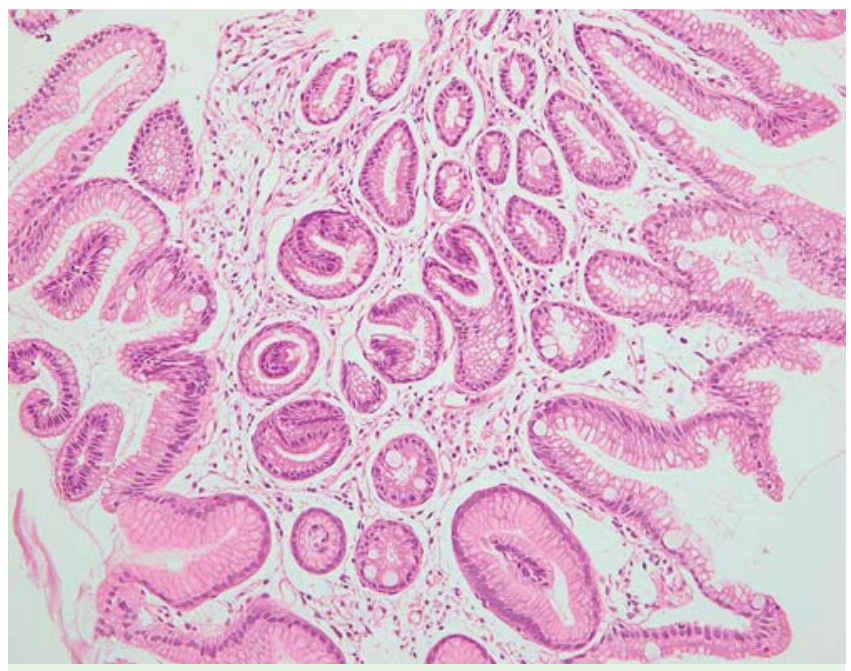

Fig. 5 The histopathologic findings in the affected mucosa showed mildly swollen epithelial cells containing intestinal metaplasia cells and interstitial edema with little inflammation.

cluded mildly swollen epithelial cells containing intestinal metaplasia cells and interstitial edema with little inflammation (○ Fig.5).

\section{Secondary outcome}

The incidence of gastric lesions (hyperplastic polyps, adenomas, cancers, erosions, or ulcers) detected in the present study did not differ significantly between the two groups ( $\bullet$ Table 1 ). In the LM group, most early gastric cancers, erosions, and ulcers appeared as well-demarcated lesions. In the placebo group, these lesions were also well demarcated when LM was subsequently administered. In addition, in subsequent detailed examinations, early gastric cancers (in four subjects) detected in the study 

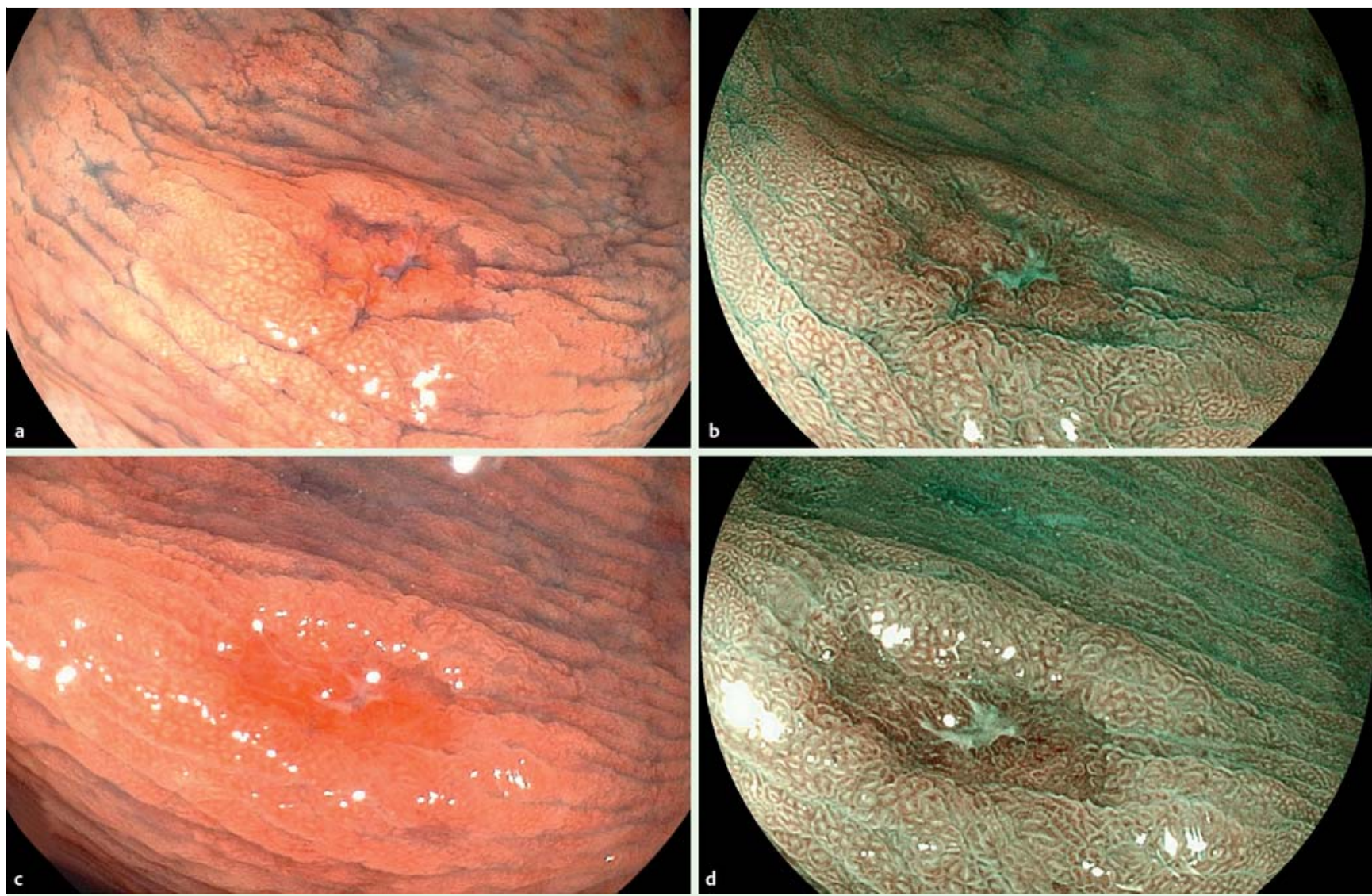

Fig. 6 Magnifying endoscopic views showing type Ilc gastric cancer. a White light endoscopic view just after the administration of placebo solution. b Blue laser imaging endoscopic view just after the administration of placebo solution. $\mathbf{c}$ White light endoscopic view just after the administration of L-Menthol (LM) solution. The llc lesion was well demarcated. It seemed that the mucosa surrounding the llc lesion became thicker than the surface of the lesion. $\mathbf{d}$ Blue laser imaging endoscopic view just after the administration of LM solution.

were also well demarcated following LM administration. This likely occurred because the mucosa surrounding the gastric cancer became thicker than the surface of the lesion ( $\bullet$ Fig. $\mathbf{6}$ ).

Gastric peristalsis was significantly suppressed in the LM group relative to the placebo group $(P<0.01)(\bullet$ Fig.7). There was no difference in antiperistaltic effect in the LM group between the subjects with and those without atrophic gastritis (proportion of subjects with no or mild peristalsis: $97 \%, 32$ of 33 subjects vs. $93.8 \%, 15$ of 16 subjects; $P=0.55$ ).

\section{Safety}

No serious adverse events, including anaphylactic reaction and symptomatic mucosal injury, occurred during the study.

\section{Discussion}

\section{$\nabla$}

The present study showed that LM sprayed on the distal stomach changed the superficial gastric mucosa into edematous mucosa, although these changes were reversible and harmless. The LM dose used in this study has been reported to be appropriate and safe for antiperistalsis during EGD [10]. However, the oral administration of higher doses causes significant adverse effects [12], and even if it is administered topically, frequent contact with LM has been reported to cause oropharyngolaryngeal injury $[13,14]$ or skin injury [15]. These topical injuries due to LM are described as "chemical burns." This may mean that direct contact between LM and the mucosal membrane causes a burnlike in-

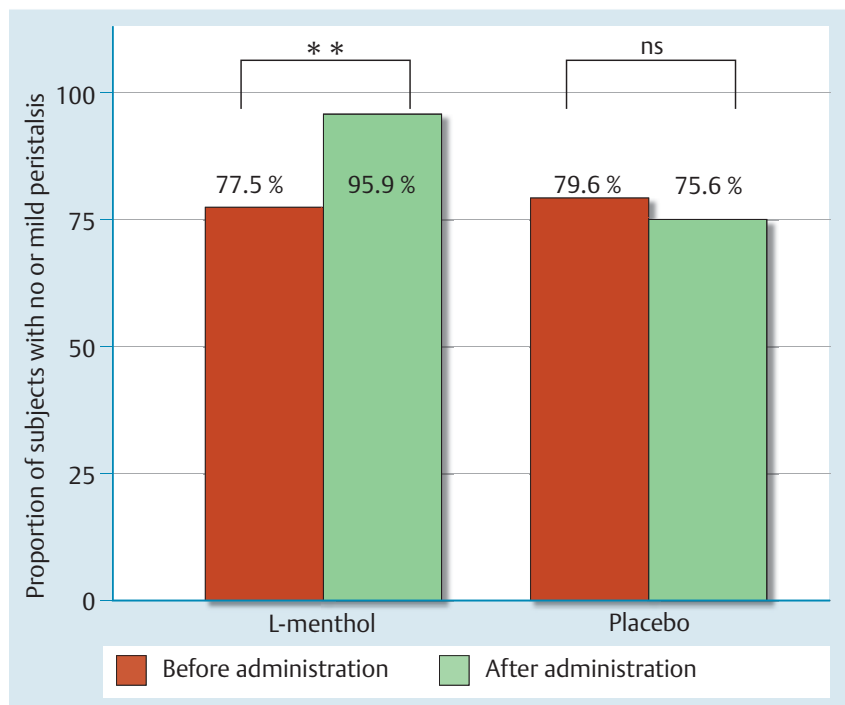

Fig.7 The proportion of subjects with no or mild peristalsis in each group. Gastric peristalsis was significantly suppressed in the L-menthol group relative to the placebo group $\left({ }^{* *}: P<0.01\right.$; ns: not significant).

jury. Therefore, the specific gastric mucosal change induced by LM is likely a reversible minor chemical burn. Although it is unclear why LM provokes such morphologic changes, the appearance of these changes was associated with the presence of atrophic gastritis. The present study revealed that these changes 
were significantly provoked in subjects with atrophic gastritis and extended to the gastric body in patients with advanced atrophic gastritis. Atrophic gastric mucosa is replaced with intestinal metaplasia containing absorptive epithelial cells, which does not exist naturally in healthy gastric mucosa [16]. Therefore, we speculate that LM undergoes endocytosis by absorptive cells in atrophic gastric mucosa with intestinal metaplasia, provoking these specific changes. In the biopsy specimens of the affected mucosa in the LM group, the intestinal metaplasia cells were contained, and histopathologically, mildly swollen epithelial cells and interstitial edema with little inflammation were observed. This finding may well support the above speculation. However, there are some limitations in considering the change as solid evidence because the histologic examinations were not exhaustive. Conversely, since the antiperistaltic effect of LM has been reported to relax gastrointestinal smooth muscle [17], the possible mucosal changes may be caused by the extension of muscle fibers. However, because muscular relaxation extends the gastric mucosa, this would be expected to show a taut surface rather than rugae.

Limited information is available regarding impact of LM on gastric cancer diagnosis $[8,18]$. Hikichi and colleagues [8] reported that the intragastric administration of LM was helpful in identifying the demarcation line of gastric cancers because LM-induced edema appeared in the perilesional nonpathologic gastric mucosa. The present study showed that the specific mucosal change appeared in the atrophic gastric mucosa and suggested that this change may be the result of LM absorption into intestinal metaplasia cells. This finding may indicate that the intragastric administration of LM facilitates the detection or demarcation of gastric lesions without intestinal metaplasia - for example, gastric erosion, gastric ulcer, and gastric cancer. This method may be useful particularly for gastric cancer screening because the background gastric mucosa of gastric cancer is primarily atrophic gastritis with intestinal metaplasia. However, there was no significant difference between the two groups in the number of endoscopic findings despite the effect on peristalsis. Whether LM facilitates the detection of gastric lesions could be a subject for further study.

This aforementioned phenomenon induced by LM is similar to the changes observed during dynamic chemical magnifying endoscopy with the acetic acid method. The acetic acid method is reported not only to enhance cancer margins but also to estimate the grade of malignancy endoscopically [19]. In this study, we were unable to distinguish between histologic differences of gastric cancer while enhancing cancer margins. However, intestinal-type gastric cancer confined to the glandular structures (low-grade dysplasia) may become edematous with LM because it has a partial residual function of intestinal metaplasia. Therefore, the intragastric administration of LM might be used as an alternative to the acetic acid method, following further research. The frequency of the antiperistaltic effect in this study was higher than that in previous reports. The frequency of gastric aperistalsis before LM administration was more than $70 \%$, which was comparable with that observed after the administration of LM in previous reports $[2,3,10]$. The reason for this difference is likely that all subjects in the present study underwent EGD via the transnasal approach. Because transnasal EGD provokes the gagging reflex less often, vigorous peristalsis triggered by emesis seems to be less frequently induced. In addition, because transnasal EGD simultaneously reduces both sympathetic and parasympathetic activity [9], gastric peristalsis may be suppressed. This result may indicate that antiperistaltic agents are not always necessary when transnasal EGD is performed. It is beneficial for the detection of smaller lesions on screening endoscopy that transnasal EGD with LM administration suppresses gastric peristalsis safely and almost completely. Although it is of concern that an ultrathin endoscope has low resolution, we consider that it is sufficient to detect small gastric lesions or ascertain specific mucosal changes, as recently developed ultrathin endoscopes have better image resolution.

The present study has several limitations. It has an open-label design and was performed by a single endoscopist at a single center. The difference in the effect of LM between the two groups was evaluated according to subjective endoscopic findings. Indeed, in the placebo group, the proportion of subjects with specific mucosal changes $(12.2 \%)$ was different from that expected $(30 \%)$. We propose further research based on objective outcomes, such as pathologic findings.

\section{Conclusion}

$\nabla$

LM changes superficial gastric mucosa into edematous mucosa. This occurs more frequently in atrophic gastric mucosa and less in pathologic lesions. LM may facilitate the demarcation of pathologic gastric lesions without intestinal metaplasia because of the effect of LM on the surrounding mucosa.

\section{Competing interests: None}

\section{References}

1 Leung WK, Wu MS, Kakugawa $Y$ et al. Screening for gastric cancer in Asia: current evidence and practice. Lancet Oncol 2008; 9: 279-287

2 Hiki N, Kaminishi M, Yasuda K et al. Antiperistaltic effect and safety of L-menthol sprayed on the gastric mucosa for upper GI endoscopy: a phase III, multicenter, randomized, double-blind, placebo-controlled study. Gastrointest Endosc 2011; 73: 932 -941

3 Hiki N, Kaminishi M, Tanabe S et al. An open-label, single-arm study assessing the efficacy and safety of L-menthol sprayed onto the gastric mucosa during upper gastrointestinal endoscopy. J Gastroenterol 2011; 46: 873-882

4 Katoh K, Nomura M, Iga A et al. Comparison of gastric peristalsis inhibition by scopolamine butylbromide and glucagon: evaluation by electrogastrography and analysis of heart rate variability. J Gastroenterol 2003; 38: 629-635

5 Hashimoto T, Adachi K, Ishimura $N$ et al. Safety and efficacy of glucagon as a premedication for upper gastrointestinal endoscopy - a comparative study with butyl scopolamine bromide. Aliment Pharmacol Ther 2002; 16: $111-118$

6 Hiki N, Kurosaka H, Tatsutomi $Y$ et al. Peppermint oil reduces gastric spasm during upper endoscopy: a randomized, double-blind, doubledummy controlled trial. Gastrointest Endosc 2003; 57: 475 - 482

7 Imagawa A, Hata H, Nakatsu $M$ et al. Peppermint oil solution is useful as an antispasmodic drug for esophagogastroduodenoscopy, especially for elderly patients. Dig Dis Sci 2012; 57: 2379-2384

8 Hikichi T, Irisawa A, Sato $M$ et al. Utility of peppermint oil for endoscopic diagnosis of gastric tumors. Fukushima J Med Sci 2011; 57: $60-65$

9 Mori A, Ohashi $N$, Tatebe $N$ et al. Autonomic nervous function in upper gastrointestinal endoscopy: a prospective randomized comparison between transnasal and oral procedures. J Gastroenterol 2008; 43: 38 44

10 Hiki N, Kaminishi M, Yasuda K et al. Multicenter phase II randomized study evaluating dose-response of antiperistaltic effect of L-menthol sprayed onto the gastric mucosa for upper gastrointestinal endoscopy. Dig Endosc 2012; 24: 79-86

11 Kaminishi M, Yamaguchi H, Nomura S et al. Endoscopic classification of chronic gastritis based on the results of a pilot study by research society for gastritis. Dig Endosc 2002; 14: 138-151 
12 Kligler B, Chaudhary S. Peppermint oil. Am Fam Physician 2007; 75: $1027-1030$

13 Moghadam BK, Gier R, Thurlow T. Extensive oral mucosal ulcerations caused by misuse of a commercial mouthwash. Cutis 1999; 64: 131 134

14 Tamir S, Davidovich Z, Attal P et al. Peppermint oil chemical burn. Otolaryngol Head Neck Surg 2005; 133: $801-802$

15 Parys BT. Chemical burns resulting from contact with peppermint oil mar: a case report. Burns Incl Therm Inj 1983; 9: 374-375

16 Leung WK, Sung JJ. Review article: intestinal metaplasia and gastric carcinogenesis. Aliment Pharmacol Ther 2002; 16: 1209-1216
17 Hills JM, Aaronson PI. The mechanism of action of peppermint oil on gastrointestinal smooth muscle. An analysis using patch clamp electrophysiology and isolated tissue pharmacology in rabbit and guinea pig. Gastroenterology 1991; 101: 55-65

18 Imagawa A, Takeuchi K, Yoshida Y et al. The evaluation of effectiveness and safety of peppermint oil solution as antispasmodic agent during endoscopic submucosal dissection [in Japanese with English abstract]. Gastroenterol Endosc 2012; 54: 3783-3789

19 Yagi K, Aruga Y, Nakamura A et al. The study of dynamic chemical magnifying endoscopy in gastric neoplasia. Gastrointest Endosc 2005; 62: $963-969$ 